\title{
DISORDERING OF InGaN/GaN SUPERLATTICES AFTER HIGH-PRESSURE ANNEALING
}

\author{
M.D. McCluskey*, L.T. Romano**, B.S. Krusor**, D. Hofstetter**, D.P. Bour**, M. Kneissl**, \\ N.M. Johnson**, T. Suski***, J. Jun*** \\ *Department of Physics, Washington State University, Pullman, WA 99164-2814 \\ **Xerox PARC, 3333 Coyote Hill Rd., Palo Alto, CA 94304 \\ *** Unipress, Ul. Sokolowska 29, 01-142 Warsaw, Poland
}

Cite this article as: MRS Internet J. Nitride Semicond. Res. 4S1, G3.42 (1999)

\begin{abstract}
Interdiffusion of In and $\mathrm{Ga}$ is observed in InGaN multiple-quantum-well superlattices for annealing temperatures of 1250 to $1400^{\circ} \mathrm{C}$. Hydrostatic pressures of up to $15 \mathrm{kbar}$ were applied during the annealing treatments to prevent decomposition of the $\mathrm{InGaN}$ and $\mathrm{GaN}$. In as-grown material, x-ray diffraction spectra show InGaN superlattice peaks up to the fourth order. After annealing at $1400^{\circ} \mathrm{C}$ for $15 \mathrm{~min}$, only the zero-order InGaN peak is observed, a result of compositional disordering of the superlattice. Composition profiles from secondary ion mass spectrometry indicate significant diffusion of $\mathrm{Mg}$ from the $p$-type $\mathrm{GaN}$ layer into the quantum well region. This $\mathrm{Mg}$ diffusion may lead to an enhancement of superlattice disordering. For annealing temperatures between 1250 and $1300^{\circ} \mathrm{C}$, a blue shift of the InGaN spontaneous emission peak is observed, consistent with interdiffusion of In and $\mathrm{Ga}$ in the quantum-well region.
\end{abstract}

\section{INTRODUCTION}

The development of blue light-emitting diodes (LEDs) [1] and laser diodes (LDs) [2] has focused a great deal of research activity on GaN-based III-V nitrides. The band gaps of $\operatorname{In}_{x} \operatorname{Ga}_{1-x} \mathrm{~N}$ alloys cover a wide spectral range, from red $(\mathrm{InN})$ to $\mathrm{UV}(\mathrm{GaN})$, making this alloy system ideal for optoelectronic applications [3]. The diffusion characteristics of host and impurity atoms in $\mathrm{In}_{x} \mathrm{Ga}_{1-x} \mathrm{~N}$ alloys are of considerable interest [4]. In this paper, results from structural and optical studies of In-Ga interdiffusion in InGaN/GaN multiple quantum wells (MQWs) are presented.

While compositional disordering of superlattices within the InAlGaAs materials system has been extensively studied [5,6], superlattice disordering in III-nitrides has only recently been observed [4]. The interdiffusion of $\mathrm{In}$ and $\mathrm{Ga}$ in $\mathrm{In}_{x} \mathrm{Ga}_{1-x} \mathrm{~N}$ alloys is complicated by the immiscibility of InN and $\mathrm{GaN}$ [7], which can result in phase separation in thick InGaN layers [8] and MQW structures [9]. In this study, we have investigated the diffusion of In and $\mathrm{Ga}$ in annealed $\mathrm{In}_{0.18} \mathrm{Ga}_{0.82} \mathrm{~N} / \mathrm{GaN}$ MQW structures. We observe quantum-well superlattice disordering, with no phase separation, for annealing temperatures from 1250 to $1400^{\circ} \mathrm{C}$. 


\section{EXPERIMENTAL DETAILS}

The MQW structures were grown by metalorganic chemical vapor deposition (MOCVD). The structures consist of a $1 \mu \mathrm{m}$ GaN:Mg layer, a 20 period superlattice of $16 \AA \operatorname{In}_{0.18} G_{0.82} \mathrm{~N}$ well / $64 \AA$ GaN barrier, and a $4 \mu \mathrm{m} \mathrm{GaN}$ :Si layer on a sapphire substrate. The thickness of the well plus barrier was determined by the spacing between satellite peaks in the X-ray diffraction (XRD) spectrum. A barrier-to-well thickness ratio of approximately 4:1 was measured with transmission electron microscopy (TEM). The In concentration in the InGaN quantum wells was determined by Rutherford backscattering spectrometry (RBS), by assuming the absence of In within the GaN barriers. Since the In concentration in the barriers is nonzero, however, the estimated In concentration of $x=0.18$ should be regarded as an upper bound.

The samples were annealed at temperatures ranging from 1200 to $1400^{\circ} \mathrm{C}$ in a highpressure furnace [10]. Pressures of up to $15 \mathrm{kbar}$ were applied, with purified $\mathrm{N}_{2}$ as a pressuretransmitting medium, in order to prevent decomposition of the $\mathrm{GaN}$ and InGaN. Pieces of $\mathrm{Mg}$ were placed in the high-pressure furnace near the sample to provide a $\mathrm{Mg}$ overpressure during annealing. To ensure the quasi-equilibrium conditions for the high-pressure annealing, GaN powder was placed in the crucible. The temperature was increased from room temperature, at a rate of $1800^{\circ} \mathrm{C} / \mathrm{h}$, until it reached a point $\sim 100^{\circ} \mathrm{C}$ below the annealing temperature. At that point, the rate was reduced to $1000^{\circ} \mathrm{C} / \mathrm{h}$ until the annealing temperature was reached. After annealing for $15 \mathrm{~min}$, the temperature was decreased at a rate of $3000^{\circ} \mathrm{C} / \mathrm{h}$.

To enable optical pumping studies, the top GaN:Mg layer was etched to a thickness of $150 \mathrm{~nm}$ by chemically-assisted ion beam etching (CAIBE) after the high-pressure anneal. Optical pumping was performed with a pulsed $337-\mathrm{nm} \mathrm{N}_{2}$ laser with a peak power of $250 \mathrm{~kW}$. The laser light was focused to a $100 \mu \mathrm{m}$ wide by 4-mm long stripe. The pump beam was attenuated by inserting a variable number of 1-mm thick glass slides between the lenses and the sample. The output of the InGaN/GaN MQW sample was collected by a $30 \times$ microscope objective, focused onto a quartz fiber, and fed into an Oriel spectrometer with a resolution of 1 $\mathrm{nm}$ and a grating with 1200 lines/mm. The light was detected by a photodetector array with 1024 elements at the output slit. Intensity versus wavelength data was acquired by collecting light for up to $300 \mathrm{~s}$ in order to maximize the signal-to-noise ratio.

\section{RESULTS}

\section{$\underline{\text { X-ray diffraction }}$}

The XRD spectra for the as-grown and annealed material are shown in Fig. 1. In the asgrown spectrum, InGaN (0006) satellite diffraction peaks were observed up to the $4^{\text {th }}$ order. The satellite peaks arise from the periodicity of the quantum-well superlattice. The XRD spectrum for material that was annealed at $1200^{\circ} \mathrm{C}$ for $15 \mathrm{~min}$ (not shown) is identical to the as-grown spectrum. After annealing at a temperature of $1250^{\circ} \mathrm{C}$ for $15 \mathrm{~min}$, the intensities of the $1^{\text {st }}, 2^{\text {nd }}$, and $3^{\text {rd }}$ order peaks were reduced, and the $4^{\text {th }}$ order peak was not detected. The reduction in satellite peak intensity is consistent with interdiffusion of In and $\mathrm{Ga}$ in the MQW region, resulting in a broadening of the quantum well profiles. Previous results from XRD and transmission electron microscopy indicated that after annealing at $1400^{\circ} \mathrm{C}$ for $15 \mathrm{~min}$, the quantum well region was replaced by a relatively uniform InGaN layer [4]. 


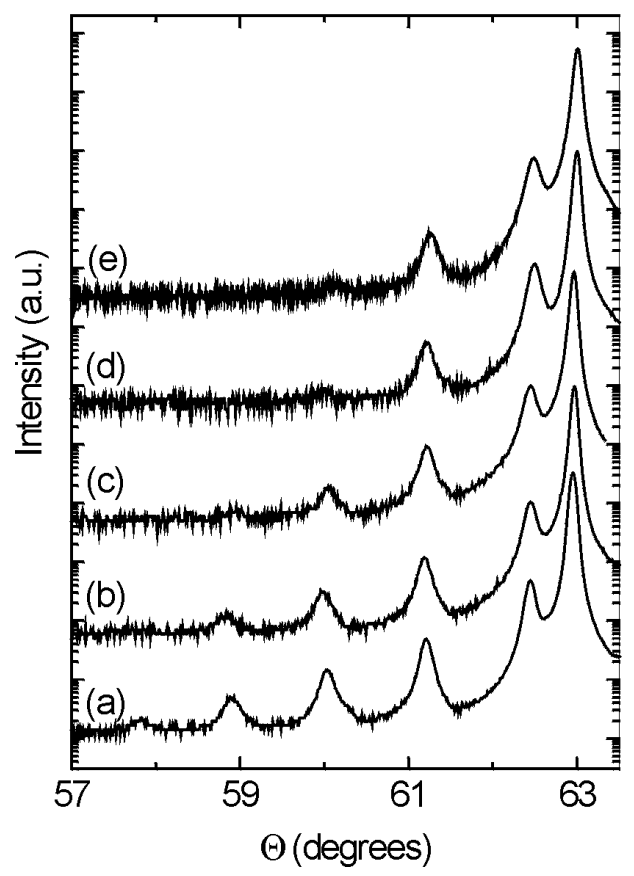

Figure 1. X-ray diffraction spectra for (0006) reflections of InGaN/GaN MQWs: (a) as-grown, and annealed for $15 \mathrm{~min}$ at (b) $1225^{\circ} \mathrm{C}$, (c) $1250^{\circ} \mathrm{C}$, (d) $1275^{\circ} \mathrm{C}$, and (e) $1300^{\circ} \mathrm{C}$.

\section{$\underline{\text { Secondary Ion Mass Spectrometry }}$}

The In and Mg concentrations were measured as a function of depth with secondary ion mass spectrometry (SIMS). The depth resolution was limited to $\sim 500 \AA$, due to surface roughness, so that the individual quantum wells were not resolved. Before annealing, the $\mathrm{Mg}$ concentration is constant to a depth of $1 \mu \mathrm{m}$, after which point it sharply decreases to a level below the detection limit $\left(\sim 10^{16} \mathrm{~cm}^{-3}\right)$. Compared to $\mathrm{Mg}$, however, In does not show significant diffusion under these annealing conditions [4].

The In-Ga interdiffusion in the MQW region is correlated with the diffusion of Mg. It is possible that the diffusion of $\mathrm{Mg}$ enhances the interdiffusion of In and $\mathrm{Ga}$ in InGaN/GaN MQWs. Impurity induced disordering was first observed in Zn-diffused AlAs/GaAs superlattices [11]. In the case of $\operatorname{In}_{x} \mathrm{Ga}_{1-x} \mathrm{As} / \mathrm{GaAs} \mathrm{MQW}$ 's with $x=0.15$, significant interdiffusion has been observed at temperatures less than $700^{\circ} \mathrm{C}$ [12]. The fact that no interdiffusion is observed in $\mathrm{In}_{x} \mathrm{Ga}_{1-x} \mathrm{~N} / \mathrm{GaN}$ MQW's for temperatures below $1300^{\circ} \mathrm{C}$ is a result of the comparatively low rates of diffusion in the III-V nitrides. Although our results are suggestive of impurity induced disordering, further studies will be required to determine the effect of impurities on the interdiffusion of In and Ga in InGaN. 


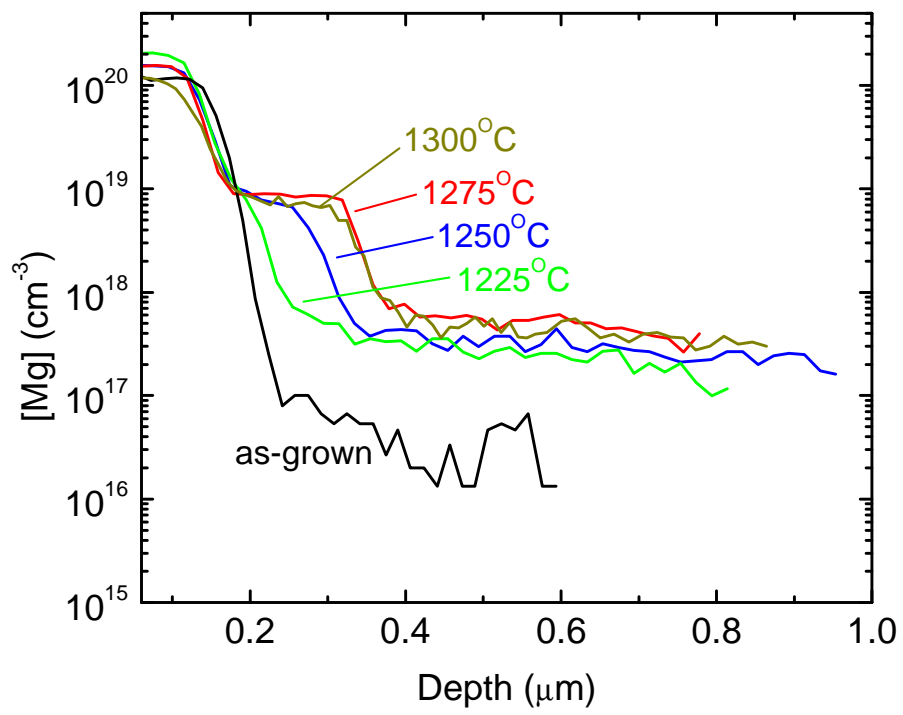

Figure 2. SIMS profiles of Mg in InGaN MQWs, for as-grown and annealed material.

\section{$\underline{\text { Spontaneous Emission }}$}

Spontaneous emission peaks for as-grown and annealed samples are shown in Fig. 3. The decrease in peak wavelength is consistent with In-Ga interdiffusion, which increases the band gap of the InGaN quantum wells [13]. The peak intensities of the annealed samples were reduced by a factor of $\sim 50$ with respect to the as-grown samples. To obtain comparable signals, larger pump intensities were used for the annealed material than for the as-grown material. While the as-grown material exhibited lasing for sufficiently high pump intensities, lasing was not observed for the annealed samples. The shift of the spontaneous emission peak is plotted in Fig. 4 for several annealing temperatures. 


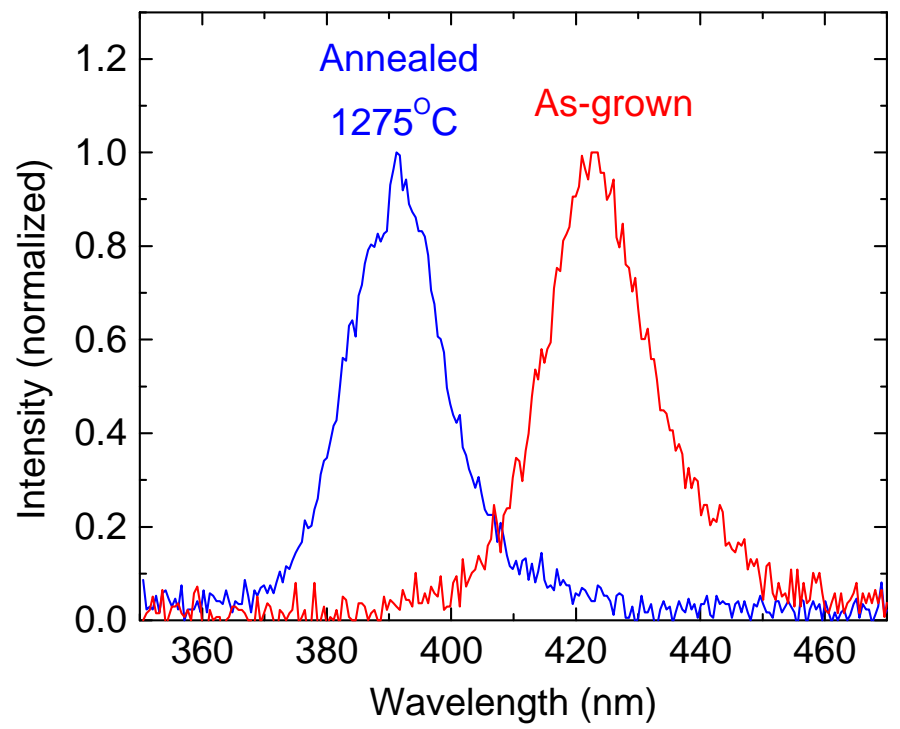

Figure 3. Emission spectra of InGaN MQWs before and after annealing.

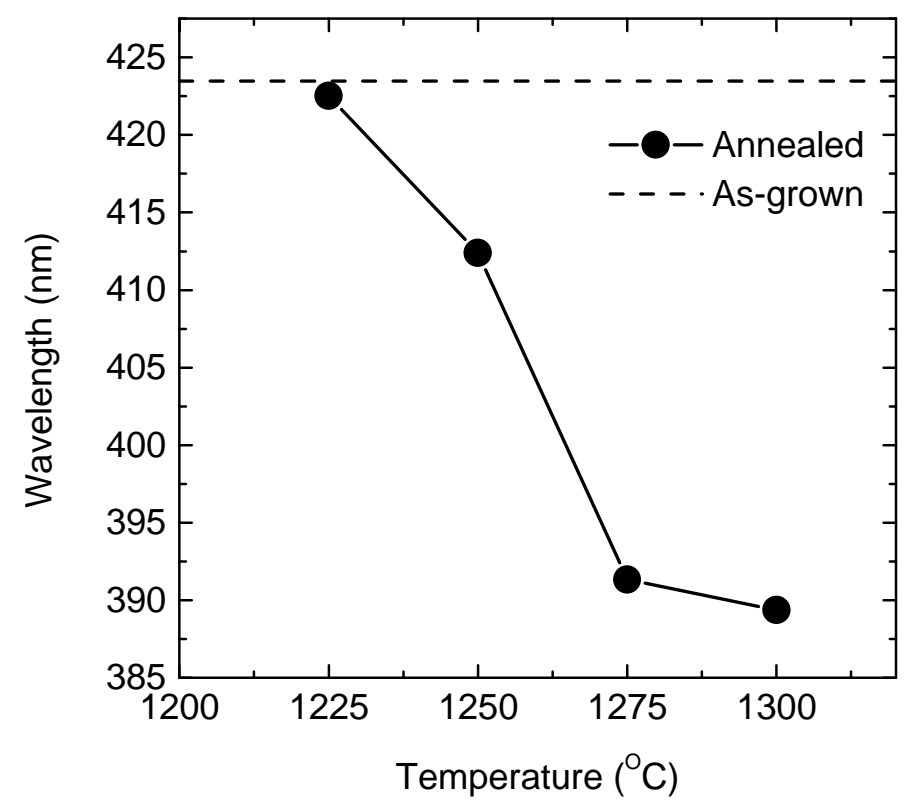

Figure 4. Spontaneous emission peak wavelengths for as-grown and annealed InGaN MQWs. 


\section{CONCLUSIONS}

In conclusion, compositional disordering has been observed in $\operatorname{In}_{x} \mathrm{Ga}_{1-x} \mathrm{~N} / \mathrm{GaN}$ MQW's with $x=0.18$. This disordering results in a blue shift of the spontaneous emission peak. Similar to the case of $\mathrm{Zn}$-diffusion in AlGaAs, it is conceivable that Mg-diffusion enhances In-Ga interdiffusion in InGaN.

\section{ACKNOWLEDGMENTS}

The authors are pleased to thank P. Floyd for helpful discussions. The work at Xerox was supported by DARPA MDA972-96-3-0014. The work at Unipress was supported by KBN 7T08A 00713.

\section{REFERENCES}

1. S. Nakamura, M. Senoh, N. Iwasa, and S. Nagahama, Jpn. J. Appl. Phys. 34, L797 (1995).

2. S. Nakamura, M. Senoh, S. Nagahama, N. Iwasa, T. Yamada, T. Matsushita, H. Kiyoko, and Y. Sugimoto, Jpn. J. Appl. Phys. 35, L74 (1996).

3. See, for example, F.A. Ponce and D.P. Bour, Nature 386, 351 (1997).

4. M.D. McCluskey, L.T. Romano, B.S. Krusor, N.M. Johnson, T. Suski, and J. Jun, Appl. Phys. Lett. 73, 1281 (1998).

5. L.L. Chang and A. Koma, Appl. Phys. Lett. 29, 138 (1976).

6. D. Hofstetter, B. Maisenhölder, and H.P. Zappe, IEEE Journal of Selected Topics in Quantum Electronics 4, 794 (1998).

7. I-hsiu Ho and G.B. Stringfellow, Appl. Phys. Lett. 69, 2701 (1996).

8. R. Singh, D. Doppalapudi, T.D. Moustakas, and L.T. Romano, Appl. Phys. Lett. 70, 1089 (1997).

9. M.D. McCluskey, L.T. Romano, B.S. Krusor, D.P. Bour, N.M. Johnson, and S. Brennan, Appl. Phys. Lett. 72, 1730 (1998).

10. T. Suski, J. Jun, M. Leszczyñski, H. Teisseyre, S. Strite, A. Rockett, A. Pelzmann, M. Kamp, and K.J. Ebeling, J. Appl. Phys. 84, 1155 (1998).

11. W.D. Laidig, N. Holonyak, Jr., M.D. Camras, K. Hess, J.J. Coleman, P.D. Dapkus, and J. Bardeen, Appl. Phys. Lett. 38, 776 (1981).

12. W.D. Laidig, J.W. Lee, P.K. Chiang, L.W. Simpson, and S.M. Bedair, J. Appl. Phys. 54, 6382 (1983).

13. K.S. Chan, E.H. Li, and M.C.Y. Chan, IEEE J. Quantum Electron. 34, 157 (1998). 